\title{
Unconventional Superconductivity in Iron-Based Superconductors in a Three-Band Model
}

\author{
D. Crivelli And A. PTOK* \\ Institute of Physics, University of Silesia, 40-007 Katowice, Poland
}

\begin{abstract}
Iron-based superconductors exhibit features of systems where the Fulde-Ferrell-Larkin-Ovchinnikov phase, a superconducting state with non-zero total momentum of the Cooper pairs, is actively sought. Experimental and theoretical evidence points strongly to the Fulde-Ferrell-Larkin-Ovchinnikov phase in these materials above the Pauli limit. In this article we discuss the ground state of iron-based superconductors near the critical magnetic field and the full $h-T$ phase diagram for pnictides in case of intra-band pairing, in a three-band model with $s_{ \pm}$ symmetry.
\end{abstract}

DOI: 10.12693 /APhysPolA.126.A-16

PACS: 74.20.Rp, 74.70.Xa, 74.25.Dw

\section{Introduction}

In '60s of the XX century, two independent groups, Fulde-Ferrell (FF) [1] and Larkin-Ovchinnikov (LO) [2], proposed a superconducting phase with oscillating order parameter $(\mathrm{OP})$ in real space. This phase, nowadays called the Fulde-Ferrell-Larkin-Ovchinnikov (FFLO) phase, is more stable than the BCS phase in low temperature and hight magnetic field regime. FF proposed a superconducting phase with one momentum $\boldsymbol{q}$ possible for the Cooper pairs, whereas LO assumed the possibility of two opposite momenta $\pm \boldsymbol{q}$ - in this case the OP in real space is proportional to $\exp (\mathrm{i} \boldsymbol{q} \cdot \boldsymbol{r})$ or $\cos (\boldsymbol{q} \cdot \boldsymbol{r})$ respectively. A non-zero total momentum of the Cooper pairs bears as a consequence the change of sign of the OP in real space and breaks the spatial symmetry of the system (this is true not only in systems with translation symmetry, but also when rotational symmetry is present [3-5]).

The FFLO phase can be expected in materials with relatively high Maki parameter $\alpha \sim H_{c 2}^{\text {orb }} / H_{c 2}^{P}$, when the orbital critical magnetic field $H_{c 2}^{\text {orb }}$ is greater than the paramagnetic critical field $H_{c 2}^{P}$. Therefore a good class of candidate to find the FFLO are heavy fermions materials (such as $\mathrm{CeCoIn}_{5}$ ) [6-12], organic superconductors [13] and quantum gases [14]. The FFLO phase can exist also in inhomogeneous systems in presence of impurities [15-17] or spin density waves [18]. Moreover, these inhomogeneities can increase the tendency system to create the FFLO phase and stabilize it in a lower magnetic field $[17,19]$. The FFLO phase can be also stabilized by pair hopping interaction [20, 21] or in system with nonstandard quasiparticles with spin-dependent mass [22-24].

Other good candidates to find the FFLO phase are iron-based superconductors (IBSC) $[25-29]$ - the characteristic feature of these chemical compounds are

\footnotetext{
*corresponding author; e-mail: aptok@mmj.pl
}

iron-arsenide layers (Fig. 1a), which imply multiband properties such as the characteristic Fermi surface (with hole- and electron-like Fermi pockets around the $(0,0)$ and $(\pi, \pi)$ point respectively, illustrated in Fig. 1b) [30-32]. IBSC are materials with high Maki parameter and anisotropic upper magnetic fields [33-40]. Experimentally a phase transition inside the superconducting state has been observed, which can be evidenced about the phase transition from convectional superconductivity to the FFLO phase [41]. These results are in agreement with theoretical expectations [29, 42, 43].

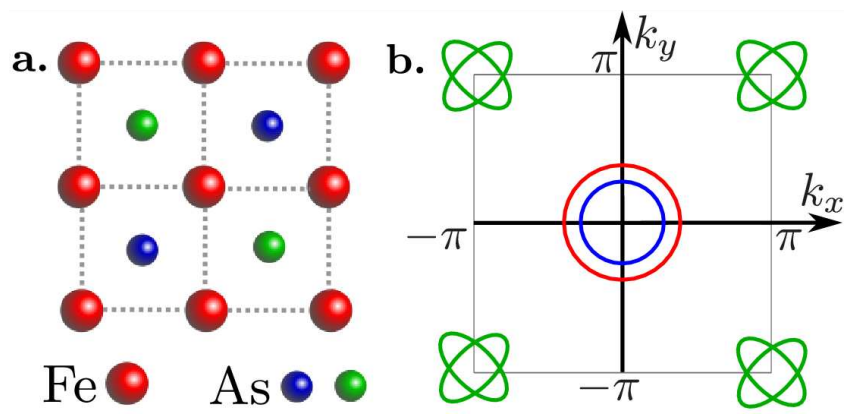

Fig. 1. (a) FeAs layer in iron-based superconductors. Fe (red dot) and As (blue and green dots) ions form a quadratic lattice. As ions are placed above (blue) or under (green) the centers of the squares formed by Fe. (b) True Fermi surface in first Brillouin zone, for two Fe ions per unit cell.

In this paper we analyze IBSC (pnictides) using the three band model proposed by Daghofer et al. [44, 45]. In Sect. 2 we describe details of theoretical calculation, in Sect. 3 we show and discuss numerical results. We summarize the results in Sect. 4. Parameters for the model are listed in Appendix (Sect. 5).

\section{Theoretical part}

The general Hamiltonian for the multi-orbital system can be written as $H=H_{0}+H_{I}$. The non-interacting part $H_{0}$ is given by 


$$
H_{0}=\sum_{\boldsymbol{k} \sigma, \alpha \beta}\left(T_{\boldsymbol{k}}^{\alpha \beta}-(\mu+\sigma h) \Delta_{\alpha, \beta}\right) c_{\boldsymbol{k} \alpha \sigma}^{\dagger} c_{\boldsymbol{k} \beta \sigma}
$$

where $c_{k \alpha \sigma}^{\dagger}\left(c_{\boldsymbol{k} \alpha \sigma}\right)$ is the creation (annihilation) operator for a spin $\sigma$ electron of momentum $k$ in the orbital $\alpha$. Hopping matrix elements $T_{\boldsymbol{k}}^{\alpha \beta}$ are given by the effective tight-binding model of the two-dimensional FeAs planes in the given model (see Appendix). Integer $\alpha$ and $\beta$ label the orbitals. Band structure of the FeAs system can be reconstructed by diagonalization of the Hamiltonian $H_{0}$ :

$$
H_{0}^{\prime}=\sum_{\boldsymbol{k} \varepsilon \sigma} E_{\boldsymbol{k} \varepsilon \sigma} d_{\boldsymbol{k} \varepsilon \sigma}^{\dagger} d_{\boldsymbol{k} \varepsilon \sigma} .
$$

$\mu$ is the chemical potential, changing the average number of particles in the system $n=\frac{1}{N} \sum_{\boldsymbol{k} \alpha \sigma} c_{\boldsymbol{k} \alpha \sigma}^{\dagger} c_{\boldsymbol{k} \alpha \sigma}=$ $\frac{1}{N} \sum_{k \alpha \sigma} d_{k \alpha \sigma}^{\dagger} d_{\boldsymbol{k} \alpha \sigma}$, where $N$ is the number of lattice site. $h$ is the external magnetic field parallel to lattice. $\varepsilon$ labels the bands.

We introduce a superconducting pairing between quasi-particles in bands $\varepsilon$. In absence of interband pairing or when it is weak [46], we can effectively describe superconductivity in the FFLO phase by the Hamiltonian

$$
H_{\mathrm{SC}}^{\prime}=\sum_{\varepsilon \boldsymbol{k}}\left(\Delta_{\varepsilon \boldsymbol{k}} d_{\varepsilon \boldsymbol{k} \uparrow}^{\dagger} d_{\varepsilon,-\boldsymbol{k}+\boldsymbol{q}_{\varepsilon} \downarrow}^{\dagger}+\text { H.c. }\right) \text {, }
$$

where $\Delta_{\varepsilon \boldsymbol{k}}=\Delta_{\varepsilon} \eta(\boldsymbol{k})$ is the amplitude of the OP for the Cooper pairs with total momentum $\boldsymbol{q}_{\varepsilon}$. The structure factor is given by $\eta(\boldsymbol{k})=4 \cos \left(k_{x}\right) \cos \left(k_{y}\right)$ for $s_{ \pm}$-wave symmetry of the OP [28]. As we see, in case of intra-band pairing we have formally an $n$-band system described by the total Hamiltonian $H=H_{0}^{\prime}+H_{\mathrm{SC}}^{\prime}$, with $n$ independent bands $\varepsilon$. Using the Bogoliubov transformation we can find a final fermions basis $\Gamma_{\varepsilon \boldsymbol{k}}=\left(\gamma_{\varepsilon \boldsymbol{k} \uparrow}, \gamma_{\varepsilon,-\boldsymbol{k} \downarrow}\right)^{\mathrm{T}}$, describing the quasi-particle excitation in the superconducting state

$$
H=\sum_{\varepsilon \boldsymbol{k} \tau} \bar{E}_{\varepsilon \boldsymbol{k} \tau} \gamma_{\varepsilon \boldsymbol{k} \tau}^{\dagger} \gamma_{\varepsilon \boldsymbol{k} \tau}+\text { const }
$$

with

$$
\begin{aligned}
& \bar{E}_{\varepsilon \boldsymbol{k} \tau}=\frac{E_{\varepsilon \boldsymbol{k} \uparrow}-E_{\varepsilon,-\boldsymbol{k}+\boldsymbol{q} \downarrow}}{2} \\
&+\tau \sqrt{\left(\frac{E_{\varepsilon \boldsymbol{k} \uparrow}+E_{\varepsilon,-\boldsymbol{k}+\boldsymbol{q} \downarrow}}{2}\right)^{2}+\left|\Delta_{\varepsilon \boldsymbol{k}}\right|^{2}}
\end{aligned}
$$

where $\tau= \pm$. Total free energy is given by $\Omega=\sum_{\varepsilon} \Omega_{\varepsilon}$, where

$$
\begin{aligned}
& \Omega_{\varepsilon}=-k_{\mathrm{B}} T \sum_{\boldsymbol{k} \tau} \ln \left(1+\exp \left(-\beta \bar{E}_{\varepsilon \boldsymbol{k} \tau}\right)\right) \\
& \quad+\sum_{\boldsymbol{k}}\left(E_{\varepsilon \boldsymbol{k} \downarrow}-\frac{\left|\Delta_{\varepsilon \boldsymbol{k}}\right|^{2}}{V_{\varepsilon}}\right)
\end{aligned}
$$

is the free energy in band $\varepsilon$ in the presence of effective interaction intensity $V_{\varepsilon}$. The ground state for fixed $h$ and $T$ can be found by minimizing the free energy with respect to the OPs.

\section{Numerical results}

Numerical calculations were carried out for a square lattice $N_{X} \times N_{Y}=2000 \times 2000$ with periodic boundary conditions. First, the effective pairing intra-band potential $V_{\varepsilon}$ has been determined for every band, in case of $s_{ \pm}$symmetry of the order parameter - to find its value we seek the disappearance of the superconducting BCS phase in each band at the same critical magnetic field $h_{\mathrm{C}}^{\mathrm{BCS}}=0.005 \mathrm{eV}$ (and temperature $k_{\mathrm{B}} T=10^{-5} \mathrm{eV}$ ). Secondly, we determine the $h-T$ phase diagram for those fixed values.

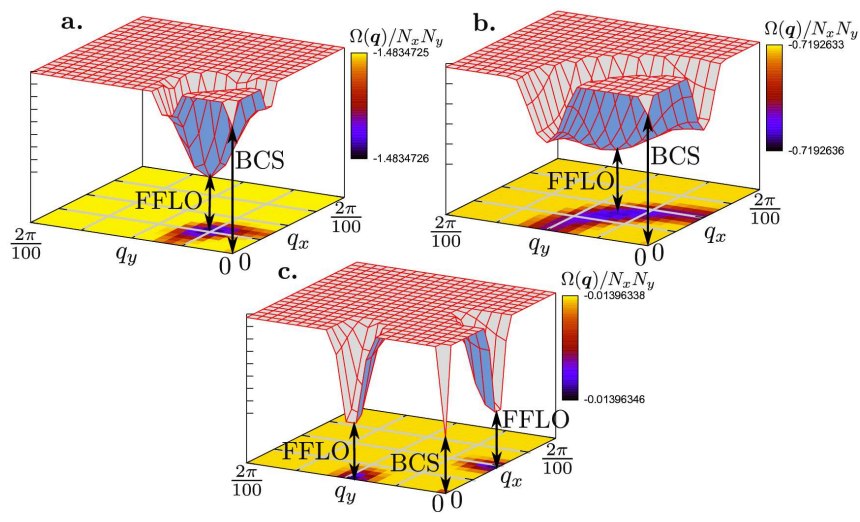

Fig. 2. The free energy per site $\Omega_{\varepsilon}(\boldsymbol{q}) / N_{x} N_{y}$ for $s_{ \pm}$ symmetry, for different values of the Cooper pair momentum $\boldsymbol{q}$, showing the location of the minima and indicating the existence of different phases. Results for $h \simeq 0.005 \mathrm{eV}=h_{\mathrm{C}}^{\mathrm{BCS}}$ and temperature $k_{\mathrm{B}} T=10^{-5} \mathrm{eV}$.

\subsection{Ground state at the BCS critical magnetic field}

To determine the $h-T$ phase diagram with $V_{\varepsilon}$ fixed, we vary the total momentum of the Cooper pairs $\boldsymbol{q}$ to find the ground state. Results for magnetic field $h \sim h_{\mathrm{C}}^{\mathrm{BCS}}$ and temperature $k_{\mathrm{B}} T=10^{-5} \mathrm{eV}$ are shown in Fig. 2 . As we see for every band and $\boldsymbol{q}=0$, there exists a local minimum of the free energy $\Omega_{\varepsilon}(\boldsymbol{q})$ corresponding to the $\mathrm{BCS}$ phase. However, we find the true ground state by the global minimum, which is attained for $\boldsymbol{q} \neq 0$. For the first two bands $(\varepsilon=1,2-$ parts (a) and (b), respectively) the ground state can be found for four equivalent total momenta $\boldsymbol{q}_{1,2}$ in directions $[1, \pm 1]$. In the third band ( $\varepsilon=3$ - part (c)) the global minimum also exists at non-zero total momentum of the Cooper pairs, but in direction $[0,1]$ or $[1,0]$. This result is in agreement with other theoretical results for pnictides in a minimal two-band model $[28,29]$ and one-band heavy fermions systems [12, 19, 21 (first paper), 47], where the FFLO phase exhibits precisely this direction of the momentum.

\subsection{Phase diagram $h-T$}

For fixed values of the effective pairing potential $V_{\varepsilon}$, we find the $h-T$ phase diagram for each band, as shown in Fig. 3. The region of the BCS phase on the phase diagram 


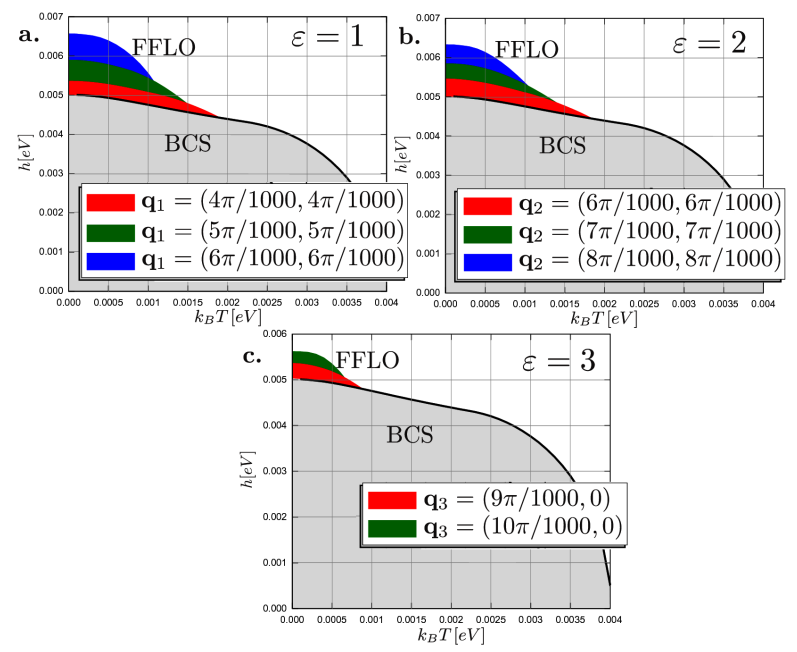

Fig. 3. $h-T$ phase diagram for given effective pairing potential $V_{\varepsilon}$. Grey area shows region of existing BCS phase. Lines mark the phase transitions. Colors (red, green and blue) mark the regions of the FFLO phase with different values of the total momentum of the Cooper pairs $\boldsymbol{q}_{\varepsilon}$.

has a typical form. Above the critical magnetic field for BCS phase and at low temperatures, the FFLO phase can form (cyan region in Fig. 3). In the first two bands, the critical magnetic field of the FFLO phase is bigger than in the third band (Fig. 3) - for the chosen values of $V_{\varepsilon}$ this difference is approximately equal to $\frac{1}{5} h_{\mathrm{C}}^{\mathrm{BCS}}$.

\subsection{Total momentum of the Cooper pairs}

Minimization of the free energy gives the total momentum of the Cooper pairs (shown in Fig. 2 in magnetic field near $\left.h_{\mathrm{C}}^{\mathrm{BCS}}\right)$. Its value $\left|\mathbf{q}_{\varepsilon}\right|$ is higher for the first band than for the second and third bands. However an increase in the magnetic field raises the total momentum magnitude (red, green and blue areas in Fig. 3), as in the IBSC two-band model [29].

In every band the critical magnetic fields of the phase transition from the FFLO phase to normal state $h_{\mathrm{C}}^{\mathrm{FFLO}}(T)$ are different. Consequence of this are the observed multiple transitions inside the FFLO area of the phase diagram, associated with changes in the modules of total momentum of the Cooper pairs $\left|\boldsymbol{q}_{\varepsilon}\right|$. Moreover this leads to amplitude modulation of the order parameter in real space, in agreement with the results in the two-band case $[29,42,43]$. To observe this feature would be an experimental check of the existence of the FFLO phase in these materials [41], since we expect more than one phase transition to exist, associated with disappearance of the FFLO phase in selected bands when increasing the external magnetic field $h$.

\section{Summary}

Using the three-band model proposed by Daghofer et al. $[44,45]$ we make a case for the FFLO phase in iron- based superconductors in presence of intra-band pairing with $s_{ \pm}$-wave symmetry. As in previous theoretical works $[28,29]$ we show that the ground state of pnictides, above the critical magnetic field of BCS phase and in low temperature, is an unconventional superconductor of the FFLO type. The full phase diagram has been obtained on lattices of thermodynamically relevant sizes, marking the typical area of the BCS phase and how the FFLO can be found beyond its borders, in regimes detrimental to the existence of BCS superconductivity. Consequence of this is the amplitude modulation of the order parameter in real space and multiple phase transitions, in agreement with the literature $[29,42,43]$.

\section{Appendix \\ Three-orbital model Daghofer et al. [44]}

This model of IBSC was proposed by Daghofer et al. in Ref. [44] and improved in Ref. [45]. Beyond $d_{x z}$ and $d_{y z}$ orbitals the model also accounts for $d_{x y}$ orbital

$$
\begin{aligned}
& T_{\boldsymbol{k}}^{11}=2 t_{2} \cos k_{x}+2 t_{1} \cos k_{y}+4 t_{3} \cos k_{x} \cos k_{y} \quad(\mathrm{~A} 1) \\
& \quad+2 t_{11}\left(\cos \left(2 k_{x}\right)-\cos \left(2 k_{y}\right)\right)+4 t_{12} \cos \left(2 k_{x}\right) \cos \left(2 k_{y}\right) \\
& T_{\boldsymbol{k}}^{22}=2 t_{1} \cos k_{x}+2 t_{2} \cos k_{y}+4 t_{3} \cos k_{x} \cos k_{y} \quad(\mathrm{~A} 2) \\
& \quad-2 t_{11}\left(\cos \left(2 k_{x}\right)-\cos \left(2 k_{y}\right)\right)+4 t_{12} \cos \left(2 k_{x}\right) \cos \left(2 k_{y}\right) \\
& T_{\boldsymbol{k}}^{33}=\epsilon_{0}+2 t_{5}\left(\cos k_{x}+\cos k_{y}\right)+4 t_{6} \cos k_{x} \cos k_{y} \\
& \quad+2 t_{9}\left(\cos \left(2 k_{x}\right)+\cos \left(2 k_{y}\right)\right) \\
& \quad+4 t_{10}\left(\cos \left(2 k_{x}\right) \cos k_{y}+\cos k_{x} \cos \left(2 k_{y}\right)\right), \\
& T_{\boldsymbol{k}}^{12}=T_{\boldsymbol{k}}^{21}=4 t_{4} \sin k_{x} \sin k_{y}, \\
& T_{\boldsymbol{k}}^{13}=\overline{\mathrm{T}}_{\boldsymbol{k}}^{31}=2 \mathrm{i} t_{7} \sin k_{x}+4 \mathrm{i} t_{8} \sin k_{x} \cos k_{y}, \\
& T_{\boldsymbol{k}}^{23}=\overline{\mathrm{T}}_{\boldsymbol{k}}^{32}=2 \mathrm{i} t_{7} \sin k_{y}+4 \mathrm{i} t_{8} \sin k_{y} \cos k_{x} .
\end{aligned}
$$

In Ref. [45] the hopping parameters in electron volts are given as: $t_{1}=-0.08, t_{2}=0.1825, t_{3}=0.08375, t_{4}=$ $-0.03, t_{5}=0.15, t_{6}=0.15, t_{7}=-0.12, t_{8}=0.06$, $t_{9}=0.0, t_{10}=-0.024, t_{11}=-0.01, t_{12}=0.0275$ and $\epsilon_{0}=0.75$. Average number of particles in the system $n=4$ is attained for $\mu=0.4748$.

\section{Acknowledgments}

D.C. acknowledges support by the FORSZT Ph.D. fellowship.

\section{References}

[1] P. Fulde, R.A. Ferrell, Phys. Rev. 135, A550 (1964).

[2] A.I. Larkin, Yu.N. Ovchinnikov, Zh. Eksp. Teor. Fiz. 47, 1136 (1964); Sov. Phys. JETP 20, 762 (1965).

[3] Y. Yanase, Phys. Rev. B 80, 220510 (R) (2009).

[4] M. Iskin, C.J. Williams, Phys. Rev. A 78, 011603 (R) (2008).

[5] A. Ptok, J. Supercond. Nov. Magn. 25, 1843 (2012). 
[6] C. Capan, A. Bianchi, R. Movshovich, A.D. Christianson, A. Malinowski, M.F. Hundley, A. Lacerda, P.G. Pagliuso, J.L. Sarrao, Phys. Rev. B 70, 134513 (2004).

[7] C.F. Miclea, M. Nicklas, D. Parker, K. Maki, J.L. Sarrao, J.D. Thompson, G. Sparn, F. Steglich, Phys. Rev. Lett. 96, 117001 (2006).

[8] A. Bianchi, R. Movshovich, C. Capan, P. G. Pagliuso, J. L. Sarrao, Phys. Rev. Lett. 91, 187004 (2003).

[9] C. Martin, C.C. Agosta, S.W. Tozer, H.A. Radovan, E.C. Palm, T.P. Murphy, J.L. Sarrao, Phys. Rev. B 71, 020503 (2005).

[10] V.F. Correa, T.P. Murphy, C. Martin, K.M. Purcell, E.C. Palm, G.M. Schmiedeshoff, J.C. Cooley, S.W. Tozer, Phys. Rev. Lett. 98, 087001 (2007).

[11] K. Kakuyanagi, M. Saitoh, K. Kumagai, S. Takashima, M. Nohara, H. Takagi, Y. Matsuda, Phys. Rev. Lett. 94, 047602 (2005).

[12] Y. Matsuda, H. Shimahara, J. Phys. Soc. Jpn. 76 , 051005 (2007).

[13] R. Lortz, Y. Wang, A. Demuer, P.H.M. Böttger, B. Bergk, G. Zwicknagl, Y. Nakazawa, J. Wosnitza, Phys. Rev. Lett. 99, 187002 (2007).

[14] R. Casalbuoni, G. Nardulli, Rev. Mod. Phys. 76, 263 (2004).

[15] Q. Wang, C.R. Hu, C.S. Ting, Phys. Rev. B 74, 212501 (2006).

[16] Q. Wang, C.R. Hu, C.S. Ting, Phys. Rev. B 75, 184515 (2007).

[17] A. Ptok, Acta Phys. Pol. A 118, 420 (2010).

[18] A. Ptok, M.M. Maśka, M. Mierzejewski, Phys. Rev. B 84, 094526 (2011).

[19] M. Mierzejewski, A. Ptok, M.M. Maśka, Phys. Rev. B 80, 174525 (2010)

[20] A. Ptok, M. Mierzejewski, Acta Phys. Pol. A 114, 209 (2008)

[21] A. Ptok, M.M. Maśka, M. Mierzejewski, J. Phys. Condens. Matter 21, 295601 (2009); K. Kapcia, S. Robaszkiewicz, R. Micnas, J. Phys. Condens. Matter 24, 215601 (2012); K. Kapcia S. Robaszkiewicz, J. Phys. Condens. Matter 25, 065603 (2013).

[22] J. Kaczmarczyk, J. Jȩdrak, J. Spałek, Acta Phys. Pol. A 118, 261 (2010).

[23] J. Kaczmarczyk, J. Spałek, J. Phys. Cond. Matter 22, 355702 (2010)

[24] M.M. Maśka, M. Mierzejewski, J. Kaczmarczyk, J. Spałek, Phys. Rev. B 82, 054509 (2010).

[25] Y. Kamihara, T. Watanabe, M. Hirano, H. Hosono, J. Am. Chem. Soc. 130, 3296 (2008).

[26] A. Gurevich, Phys. Rev. B 82, 184504 (2010).

[27] A. Gurevich, Rep. Prog. Phys. 74, 124501 (2011).

[28] A. Ptok, D. Crivelli, J. Low Temp. Phys. 172, 226 (2013).

[29] A. Ptok, Eur. Phys. J. B 87, 2 (2014).
[30] D.J. Singh, M.H. Du, Phys. Rev. Lett. 100, 237003 (2008).

[31] H. Ding, P. Richard, K. Nakayama, K. Sugawara, T. Arakane, Y. Sekiba, A. Takayama, S. Souma, T. Sato, T. Takahashi, Z. Wang, X. Dai, Z. Fang, G.F. Chen, J.L. Luo, N.L. Wang, Europhys. Lett. 83 47001 (2008).

[32] T. Kondo, A.F. Santander-Syro, O. Copie, Ch. Liu, M.E. Tillman, E.D. Mun, J. Schmalian, S.L. Bud'ko, M.A. Tanatar, P.C. Canfield, A. Kaminski, Phys. Rev. Lett. 101, 147003 (2008).

[33] G. Fuchs, S.L. Drechsler, N. Kozlova, G. Behr, A. Köhler, J. Werner, K. Nenkov, R. Klingeler, J. Hamann-Borrero, C. Hess, A. Kondrat, M. Grobosch, A. Narduzzo, M. Knupfer, J. Freudenberger, B. Büchner, L. Schultz, Phys. Rev. Lett. 101, 237003 (2008).

[34] T. Terashima, M. Kimata, H. Satsukawa, A. Harada, K. Hazama, S. Uji, H. Harima, G.F. Chen, J.L. Luo, N.L. Wang, J. Phys. Soc. Jpn. 78, 063702 (2009).

[35] N. Kurita, K. Kitagawa, K. Matsubayashi, A. Kismarahardja, E.S. Choi, J.S. Brooks, Y. Uwatoko, S. Uji, T. Terashima, J. Phys. Soc. Jpn. 80, 013706 (2011).

[36] K. Cho, H. Kim, M.A. Tanatar, Y.J. Song, Y.S. Kwon, W.A. Coniglio, C.C. Agosta, A. Gurevich, R. Prozorov, Phys. Rev. B 83, 060502(R) (2011).

[37] J.L. Zhang, L. Jiao, F.F. Balakirev, X.C. Wang, C.Q. Jin, H. Q. Yuan, Phys. Rev. B 83, 174506 (2011).

[38] S. Khim, B. Lee, J.W. Kim, E.S. Choi, G.R. Stewart K.H. Kim, Phys. Rev. B 84, 104502 (2011).

[39] Y. Liu, M.A. Tanatar, V.G. Kogan, H. Kim, T.A. Lograsso, R. Prozorov, Phys. Rev. B 87, 134513 (2013).

[40] P. Burger, F. Hardy, D. Aoki, A.E. Böhmer, R. Eder, R. Heid, T. Wolf, P. Schweiss, R. Fromknecht, M.J. Jackson, C. Paulsen, C. Meingast, Phys. Rev. B 88, 014517 (2013).

[41] D.A. Zocco, K. Grube, F. Eilers, T. Wolf, H. v. Löhneysen, Phys. Rev. Lett. 111, 057007 (2013).

[42] T. Mizushima, M. Takahashi, K. Machida, J. Phys. Soc. Jpn. 83, 023703 (2014).

[43] M. Takahashi, T. Mizushima, K. Machida, Phys. Rev. B 89, 064505 (2014).

[44] M. Daghofer, A. Nicholson, A. Moreo, E. Dagotto, Phys. Rev. B 81, 014511 (2010).

[45] M. Daghofer, A. Nicholson, A. Moreo, Phys. Rev. B 85, 184515 (2012).

[46] I.I. Mazin, Nature 464, 183 (2010). 